\title{
THE POPLITEUS MUSCLE AND THE LATERAL MENISCUS
}

\section{With a Note on the Attachment of the Medial Meniscus}

\author{
R. J. Last, LoNdon, England \\ Lecturer in Anatomy, Anatomical Curator, Bland Sutton Research Scholar, and Warden, \\ Royal College of Surgeons of England
}

Study of the anatomy of the human popliteus muscle has shown not only that many present descriptions are faulty but also that the muscle may play an essential and hitherto unrecognised part in protecting the lateral meniscus from injury. The description of the

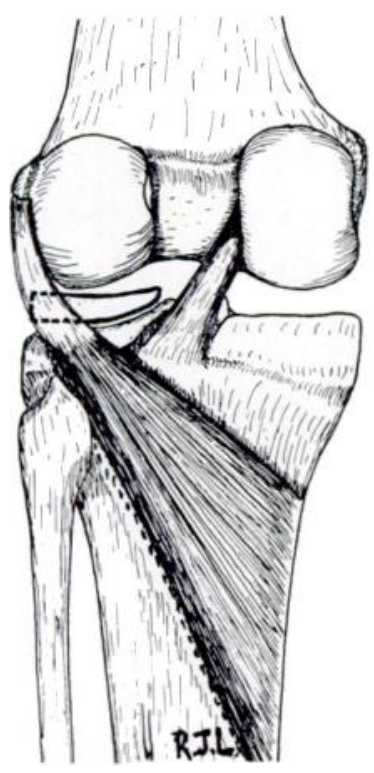

FIG. 1

The popliteus muscle as it is described in current texts. The muscle is not shown to be attached to the lateral meniscus. The ligaments of Humphry and Wrisberg are not recognised in modern terminology.

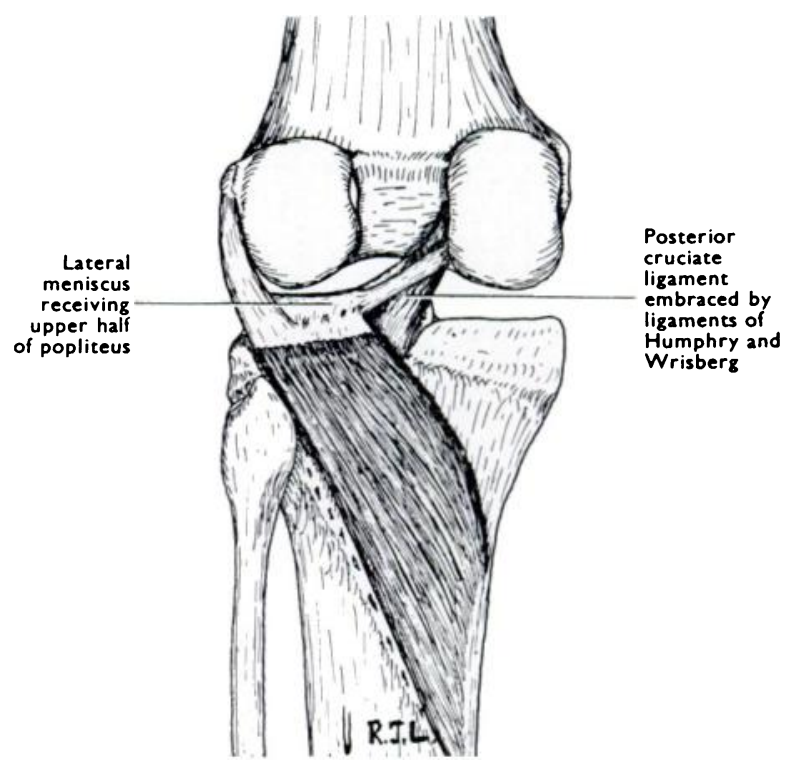

FIG. 2

In fact, the upper half of the muscle is inserted into the posterior arch of the lateral meniscus and the meniscus is attached to the femur by the ligaments of Humphry and Wrisberg. Contraction of popliteus draws the meniscus backwards during rotation of the knee.

popliteus muscle in more than a dozen current British text-books is that of a rounded tendon passing from the lateral epicondyle of the femur to a fan-shaped fleshy attachment to the popliteal surface of the tibia (Fig. 1). The tendon is sometimes said to lie free in the cavity of the knee joint, surrounded by a synovial sheath that herniates out beneath the fleshy belly as the popliteus bursa; and it has been said to bevel the posterior edge of the tibial plateau. In reality, no less than half the muscle is attached by a quadrilateral aponeurosis to the posterior arch* of the lateral meniscus and this half has no attachment to the femoral tendon; the quadrilateral aponeurosis receives direct insertion of the muscle fibres of the medial or upper half of the muscle (Fig. 2).

An occasional variant is shown in Figure 3. Here the meniscal aponeurosis arises from the deep surface of the popliteus across its whole width. In such cases the superficial fibres of the muscle converge, and are attached to the femoral tendon; while the deep fibres act by

* The term "arch" in this paper refers to the circumference of a meniscus, excluding both anterior and posterior horns. 


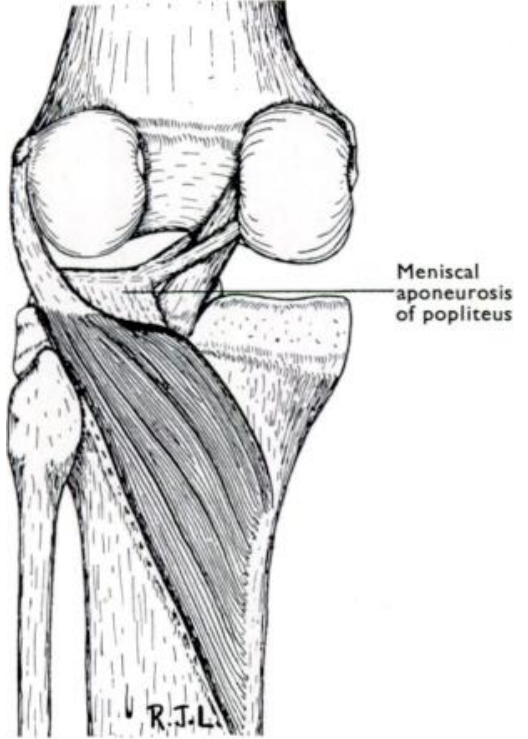

FIG. 3

An occasional variant. The superficial fibres of popliteus converge to the femoral tendon. Across the whole width of the muscle the deep fibres are attached to the aponeurosis which is inserted into the posterior arch of the lateral meniscus. Contraction of the popliteus draws the meniscus backwards during rotation of ?the

knee.

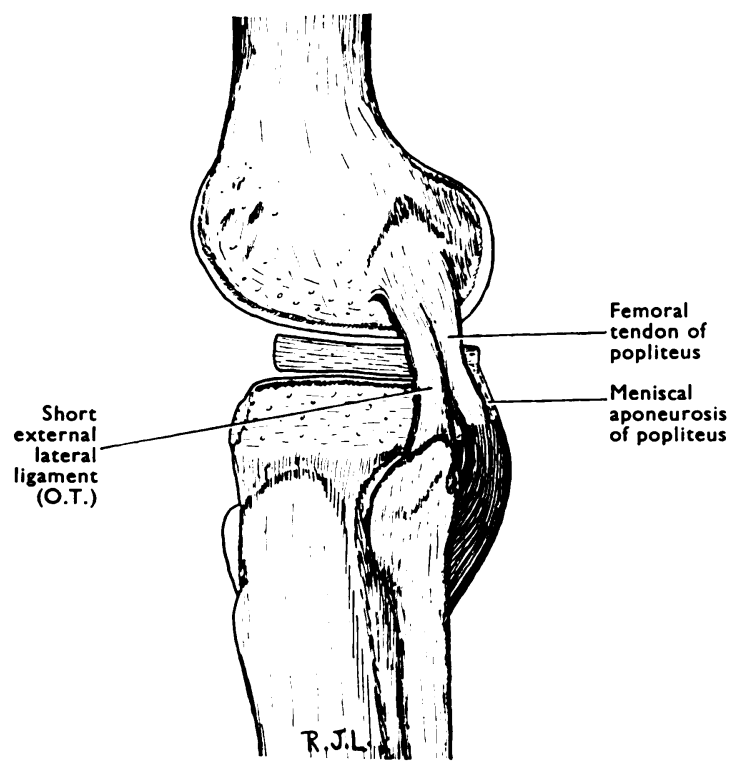

Fig. 5

The femoral tendon of popliteus. The lateral ligament of the knee is removed to show the short external lateral ligament of Old Terminology; it is fused to the anterior edge of the popliteus tendon. It is as strong as the lateral ligament; yet it is unrecognised in modern nomenclature.

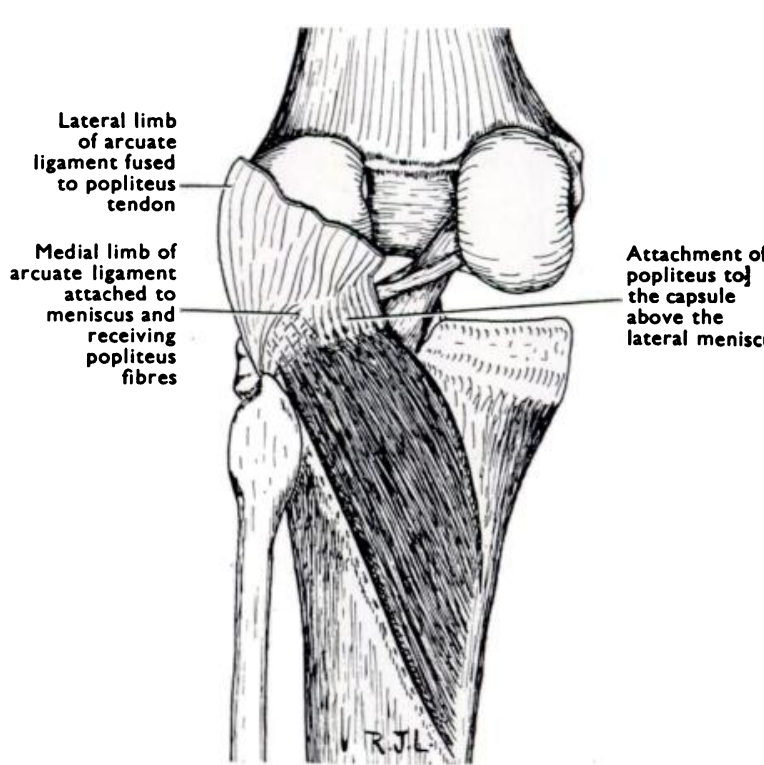

FIG. 4

Capsular attachments of popliteus. The arcuate ligament is attached to the lateral meniscus. Superficial fibres of the lower half of popliteus are inserted into it; contraction of these fibres depresses the arcuate ligament, which in turn draws the posterior arch of the meniscus downwards. The meniscal aponeurosis of popliteus shown in Figures 2 and 3 is covered by a sheet of fibres by which the popliteus is attached to the posterior part of

the capsule over the femoral condyle.

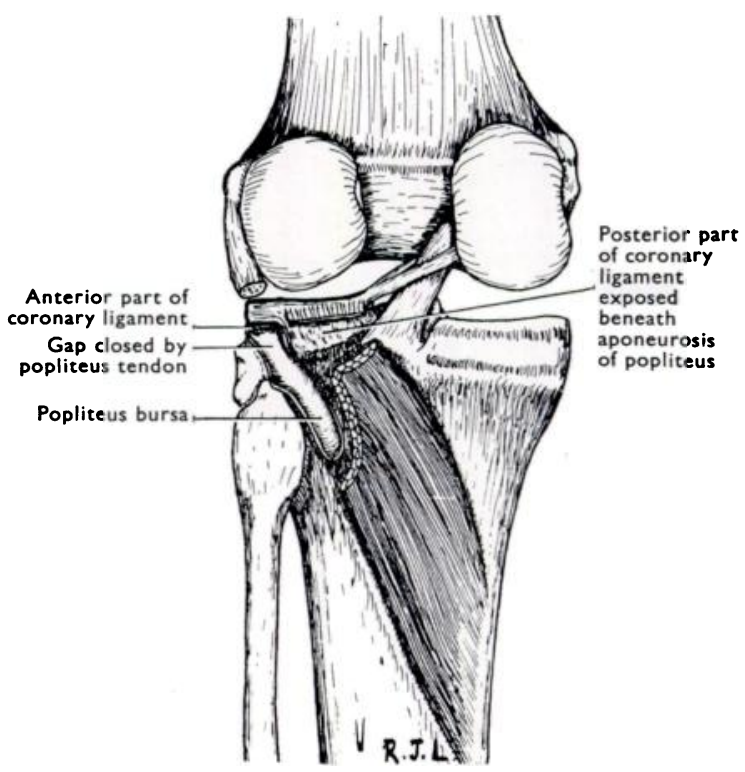

Fig. 6

Popliteus muscle divided to expose the popliteus bursa. It lies beneath the femoral part of the muscle. Division of the meniscal (upper) half of the popliteus has exposed the coronary ligament and synovial membrane; there is no popliteus bursa here.

THE JOURNAL OF BONE AND JOINT SURGERY 
way of the aponeurosis directly on the lateral meniscus. It may be that failure to notice such a deeply placed aponeurosis accounts for the error in many descriptions, because in such cases the aponeurosis can easily be cut from the deep surface of popliteus, leaving an apparently free upper border to the muscle.

In both the normal muscle and the occasional variant, fibres from the posterior surface of the muscle cover the meniscal aponeurosis and are continued up to blend with the capsule behind the lateral femoral condyle (Fig. 4). Similarly, in each case, there lies deep to the aponeurosis the weak and lax coronary ligament lined with synovial membrane attaching the inferior border of the lateral meniscus to the edge of the articular surface of the tibia. The coronary ligament is part of the true capsule of the knee joint (Fig. 6). These attachments of the popliteus to the lateral meniscus and to the capsule of the knee joint are recognised by continental authors, though none seems to have noted the functional significance of the arrangement. Such attachments are suggested too in a few English text-books but only in the illustrations where these have been reproduced from former editions and in no case can the description be found in current English texts.

The arcuate ligament is variously described. Some texts record it as arching from the fibular styloid over the popliteus muscle to blend with the oblique posterior ligament of Winslow; whereas others say that it extends from the lateral condyle of the femur to the posterior part of the capsule, being convex downwards and attached to a triangular retinaculum extending from its convexity to converge on the fibular styloid. In truth, there is at this part of the capsule such a complexity of fibres running in many directions that, by artful dissection, almost any pattern desired by the dissector could be made! By far the strongest and most consistent of these fibres form a triangular sheet that diverges upwards from the fibular styloid. The lateral limb of this mass is dense and strong, and it is attached to the femur and the popliteus tendon; while the weaker medial limb curves over the popliteus muscle, is attached to the posterior arch of the lateral meniscus and thence passes upwards to be lost on the posterior part of the capsule. The free edge of this medial limb is crescentic and beneath it the lateral, or femoral, part of the popliteus emerges to approach its tibial attachment. The attachment of the superficial fibres of the muscle to this concave edge is scarcely noted in current texts, but it is this attachment that serves to displace the arcuate band downwards and so draw the posterior arch backwards, as can be shown in an unfixed specimen by pulling downwards the superficial fibres of the lateral part of the muscle (Fig. 4). The essential facts about the functional anatomy of the arcuate ligament are that it is attached to the lateral meniscus, and, that part of the popliteus is inserted into it.

As stated, the upper or medial half of the popliteus muscle is inserted by an aponeurosis directly into the posterior arch of the lateral meniscus and has no connection with the femoral tendon of attachment. Moreover, superficial fibres of the other part are attached to the arcuate ligament and it will thus be appreciated that more than half the cross-sectional bulk of the muscle belly of the popliteus acts entirely on the posterior arch of the lateral meniscus and has no attachment at all to the femur. In the occasional variant illustrated in Figure 3 the deep half of the muscle acts on the lateral meniscus, and only the superficial half acts on the femur.

\section{TENDON OF POPLITEUS}

The posterior margin of the medial condyle of the tibia is right-angled in shape, and the articular area is confined to the upper horizontal surface of the tibia. The posterior margin of the lateral tibial condyle is rounded and the articular surface is continued downwards over the sloping area. Inspection makes it evident that the rounded bevel is caused by the posterior arch of the lateral meniscus, and not by the tendon of popliteus, which in no position of the knee joint comes into contact with this part of the tibia. The popliteus tendon makes no more than a narrow oblique groove at the postero-lateral angle of the tibial margin just above the styloid process of the fibula. Furthermore, the tendon of popliteus does not lie free

VOL. 32 B, NO. 1, FEBRUARY 1950 
within the cavity of the knee joint but is firmly attached along its lateral surface to the joint capsule which, here, is very strong and consists of the lateral limb of the arcuate ligament described above. Fused to the popliteus tendon at its femoral attachment is the upper end of the powerful "short external lateral ligament" (of Old Terminology, non-existent in modern nomenclature). The lower end of this ligament diverges from the popliteus tendon and is inserted into the styloid process and medial border of the head of the fibula. It is blended with the capsule of the joint in front of the popliteus tendon and, morphologically, has been deemed to be a thickened part of the capsule. It serves functionally to reinforce the lateral ligament of the knee joint with which indeed it is equal in strength (Fig. 5). Sometimes, however, it is absent. Only the medial surface of the popliteus tendon projects into the joint cavity, producing a ridge which is covered with synovial membrane above and below the meniscus. There is sometimes a further attachment of popliteus to the meniscus by fibrous strands directly connecting the tendon to the meniscus where the two are in contact between the synovial reflections above and below the meniscus. Usually, however, there is no such attachment and the meniscus is grooved by the passage across it of the rounded tendon. The synovial membrane below the meniscus herniates deep to the lateral or femoral part of the muscle as the popliteus bursa. There is no popliteus bursa beneath the medial or meniscal part of the muscle, for here the joint is closed by the attachment of the coronary ligament to the edge of the articular facet on the tibial plateau (Fig. 6).

\section{LIGAMENTS OF HUMPHRY AND WRISBERG}

The ligaments of Humphry and Wrisberg were well known to former generations of anatomists. Perhaps because their names have been omitted from B.N.A. and Birmingham revision nomenclature their existence goes unnoticed in many current texts; and particularly is this so of the ligament of Humphry. They are strong fibrous bands which embrace the posterior cruciate ligament and connect the posterior arch of the lateral meniscus to the medial condyle of the femur in the intercondyloid fossa (Fig. 2). In man only a small part of the posterior horn of the lateral meniscus is attached to the tibia; and in most animals the posterior horn has no tibial attachment, being held only by the ligaments of Humphry and Wrisberg to the femur. In many of these animals sudden rotary movements of the knee must be a vital necessity. On the other hand, among the Cheiroptera, the genus Pteropus (the fruit bat) have knees in which the movement of rotation is completely lacking, only flexion and extension being possible. These animals have no menisci and no popliteus muscle, which is confirmatory evidence of the purely rotatory function subserved by these structures (Parsons 1900). The function of the ligaments of Humphry and Wrisberg in holding the posterior arch in a constant relation to the rotating femur is believed by this writer to be an integral part of the mechanism of control of the lateral meniscus.

\section{ACTION OF POPLITEUS}

In flexion and extension of the knee joint, movement takes place in the upper compartment, above the menisci. It seems certain that the essential action of popliteus on the knee joint is that of rotation. In the hyperextended or so-called "locked " position of the joint it is indeed the only muscle that can have a rotatory effect. In flexion and extension of the knee joint the axis of rotation of the femoral condyle passes so nearly through the attachment of the popliteus tendon that any flexing effect of the muscle must be negligible. In the flexed position of the joint the rotators are the hamstrings, contracting alternately, and it is suggested that during such rotation the popliteus functions not merely as an accessory muscle of rotation, but essentially as a controller of the position of the lateral meniscus.

In the hyperextended or "locked" position of the knee joint it is only the popliteus muscle that has any rotatory effect because in this position any one hamstring can act on the knee joint only as a flexor. In beginning the movement of " unlocking " the hyperextended 
knee joint, the popliteus rotates the lateral condyle of the femur and draws it posteriorly on the lateral facet of the tibia. During this rotary movement the ligaments of Humphry and Wrisberg serve to draw the mobile posterior arch of the lateral meniscus in a medial direction. This movement alone would tend to flatten the posterior arch and render it liable to crushing between the femur and tibia; but simultaneously contraction of the popliteus draws the posterior arch backwards and downwards over the rounded posterior margin of the lateral tibial facet, thus maintaining its original curvature and drawing it out of harm's way. In the rotary movement of " unlocking" the hyperextended knee the movement of the lateral meniscus is minimal. In rotary movements of the flexed knee, however, the excursion of the femoral condyles on the tibial plateau is symmetrical and of considerable extent, as can be felt readily by anyone who examines his own knee. Here the range of movement of the posterior arch of the lateral meniscus is likewise considerable, and produces the bevelling of the posterior margin of the tibial condyle which has been attributed erroneously to the popliteus tendon.

This writer believes that it is not the mobility of the lateral meniscus that protects it from injury so much as the precisely controlled movements imparted by the ligaments of Humphry and Wrisberg, and the meniscal fibres of popliteus, thus ensuring that in all positions of the joint the posterior arch is held away from the millstones of the femur and tibia so that it cannot be ground between them. An attachment between the femoral tendon and meniscus, which is sometimes present, serves further to hold the meniscus in a constant relation to the rotating femoral condyle. Other fibrous bands that are occasionally seen between the lateral meniscus and the popliteus, or the posterior cruciate ligament, would act similarly. By reason of the elasticity of the meniscus the position of the anterior arch is dependent on that of the posterior arch. Thus, when the lateral condyle of the femur moves anteriorly on the tibial plateau, relaxation of the ligaments of Humphry and Wrisberg and of the popliteus muscle allows the posterior arch to glide forwards, and the anterior arch does likewise as the meniscus resumes its position of rest.

Text-books of surgery all note that injuries are sustained more often by the medial than by the lateral meniscus; and they give the proportion variously as four to one, five to one or even up to twenty to one. Many give no explanation of this fact, and those that do try to explain it assume that the lateral meniscus is protected by its mobility, though often the observation is qualified by such words as "possibly " or " probably." It is well known that mobile bodies within the joint are liable to impaction between the femur and tibia, and it is difficult to imagine why the mobile lateral meniscus should be an exception. Apart from direct violence, injuries to the menisci are produced exclusively during rotation movements of the flexed knee. The medial meniscus is fixed by its horns to the tibia, and by its whole circumference to the coronary ligament. It is attached also to part of the medial ligament of the knee joint and is thus relatively immobile on the tibia; and injury to the medial meniscus presupposes such injury as distraction, momentary stretching or rupture of the capsule or medial ligament. The lateral meniscus, on the other hand, is held but loosely by the capsule, which is here called the coronary ligament, and although its horns are connected to the tibia its whole circumference is freely mobile. When the lateral meniscus is injured it can therefore be assumed that the fault lay in the mechanism whereby the popliteus muscle normally controls its position; there may be no injury at all to the ligament or to other articular structures. In the same connection it is interesting to note that locking of the joint often occurs after rupture of the medial meniscus but seldom after injury to the lateral meniscus. In the case of the medial meniscus the torn part is uncontrolled and may be impacted between the femur and tibia; whereas in the case of the lateral meniscus the torn fragment may still be controlled by the popliteus and, though abnormally mobile, is pulled away from the femur during rotation of the knee. Mobility of loose bodies and ruptured medial menisci often causes locking of the joint by inclusion between the articular surfaces; but mobility of the lateral meniscus never causes locking because it is a controlled mobility.

VOL. $32 \mathrm{~B}$, NO. 1, FEBRUARY 1950 


\section{NOTE ON THE MOBILITY OF THE MEDIAL MENISGUS}

Of the strength of attachment of both horns of the medial meniscus to the tibia there is no doubt; a strong ligament holds each horn firmly fixed to the tibia. There seems to be some misunderstanding, however, as to the degree of fixation of the circumference of the meniscus to the articular margin of the tibia. Actually there is no firm attachment to the tibia, and the periphery of the meniscal arch is attached much more firmly to the femur than to the tibia by both superficial and deep parts of the medial ligament.

The superficial part of the medial ligament is triangular in shape. The vertical anterior border of the ligament is not attached to the meniscus; but the oblique upper and lower fibres of its posterior border converge on the meniscus and are firmly attached (Fig. 7). This area of attachment lies posterior to that of the deep part of the medial ligament described below. The fibres from the femur to the meniscus are short and they pull the arch of the

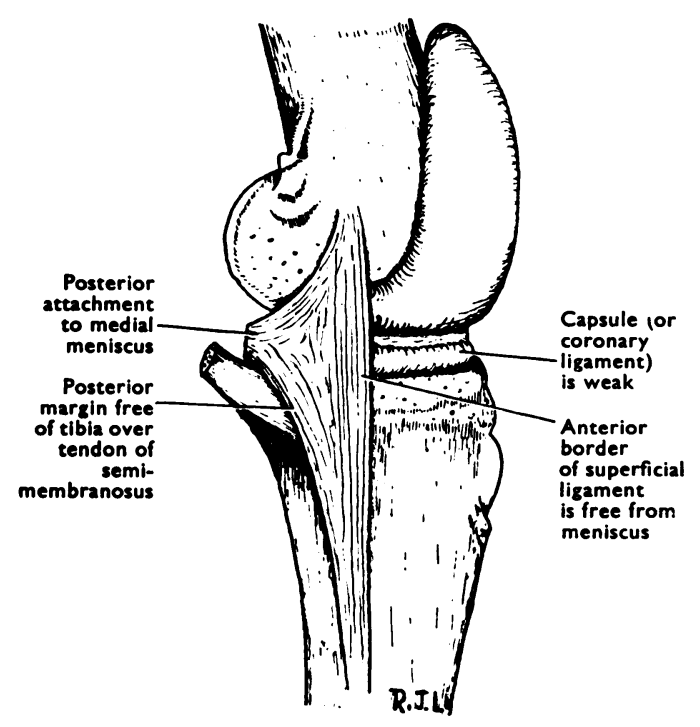

Fig. 7

The attachments of the medial meniscus. The superficial medial ligament is attached posteriorly to the medial meniscus. The short femoral fibres move with the femur; the long tibial fibres are free from the tibia over the semimembranosus expansion and the medial inferior genicular vessels. In rotation of the knee, therefore, the arch of the medial meniscus moves with the femur on the tibia.

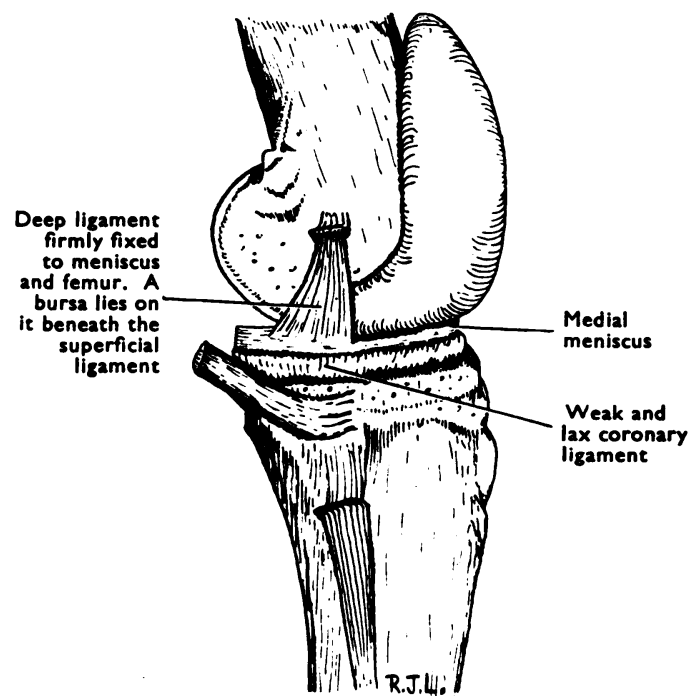

Fig. 8

The attachments of the medial meniscus. The superficial medial ligament is removed. The deep medial ligament (short internal lateral ligament of Old Terminology) is attached from femur to medial meniscus. Between the meniscus and tibia there is only lax and weak coronary ligament. The medial meniscus is not attached firmly to the tibia except at its horns. The arch of the meniscus moves with the femur during rotation of the knee.

meniscus forwards when the femur rotates laterally on the tibia. The fibres from the tibia to the meniscus are not only much longer but are separated from the upper margin of the tibia by the underlying expansion from the semimembranosus tendon and, lower down, by the medial inferior genicular vessels. In lateral rotation of the tibia on the femur these fibres therefore do not have the same effect on the arch of the meniscus; the tibial condyle slides forwards beneath the ligament and fails to move the arch of the meniscus, which remains stationary on the femur.

The deep part of the medial ligament lies hidden beneath the superficial part and is largely separated from it by an intervening bursa. It forms a strong connection between the femur and the medial meniscus, into which it is strongly inserted (Fig. 8). It is a thickening of the capsule, with which its anterior and posterior fibres blend. It is the " short internal lateral ligament" of Old Terminology. It is scarcely represented below the level of the 
medial meniscus, which is attached to the tibia here by a weak coronary ligament that is no stronger than that around the remainder of the arch. In rotation of the knee the short fibres of the deep ligament, like the oblique fibres of the superficial part, ensure that the arch of the meniscus follows the femur and moves with it on the tibia.

It is fallacious to argue that rupture of the medial meniscus is due to its fixation to the medial ligament and thus to the tibia; the arch of the meniscus is surprisingly mobile on the tibia in rotary joint movements. But the mobility, unlike that of the lateral meniscus, lacks muscular control and is purely passive. It is accompanied, moreover, by greater distortion than in the case of the lateral meniscus which is more circular and less widely fixed to the tibia at its horns. The medial meniscus sustains rupture not because it is fixed to the tibia but because its arch is mobile on the tibia, and because its movement lacks the control mechanism of the lateral meniscus.

\section{SUMMARY}

1. The movements of flexion and extension at the knee joint take place above the menisci; the movements of rotation take place below the menisci.

2. The popliteus muscle consists of two halves, one being attached by tendon to the femur, the other by aponeurosis to the lateral meniscus. The action of the muscle is to rotate the knee and retract the posterior arch of the lateral meniscus. The flexing action of the muscle is quite negligible.

3. The effects of rotation of the knee joint on the two menisci are dissimilar. The medial meniscus is distorted during rotation; its horns move with the tibia, but the intervening arch moves with the femur, and the distortion renders it liable to injury during rotation. The lateral meniscus does not become distorted during rotation; it follows the lateral condyle of the femur by reason of the attachments of the ligaments of Humphry and Wrisberg and the popliteus muscle. It is thus relatively immune from injury during rotation.

Note on dissections - The dissections upon which this article is based are in the Museum of the Royal College of Surgeons of England. Most of them have been made recently by the Prosector, D. Tompsett, Ph.D., in collaboration with the writer.

\section{REFERENCES}

Heitzmann, C. (1887): Anatomy Descriptive and Topographical. English edition by L. Heitzmann. New York: J. H. Vail \& Co. pp. 129, 201.

PArsons, F. (1900): Journal of Anatomy and Physiology, N.S. 14, 310.

Testut, L. (1928): Traité d'Anatomie Humaine. Eighth edition. Paris: G. Doin et Cie. p. 1163. 